\title{
Design and Characterization of Electrospun Polyamide Nanofiber Media for Air Filtration Applications
}

\author{
Jonas Matulevicius, ${ }^{1}$ Linas Kliucininkas, ${ }^{1}$ Dainius Martuzevicius, ${ }^{1}$ Edvinas Krugly, \\ Martynas Tichonovas, ${ }^{1}$ and Jonas Baltrusaitis ${ }^{2}$ \\ ${ }^{1}$ Department of Environmental Technology, Kaunas University of Technology, Radvilenu plentas 19, 50254 Kaunas, Lithuania \\ ${ }^{2}$ Photo Catalytic Synthesis Group, University of Twente, Meander 229, P.O. Box 217, 7500 AE Enschede, The Netherlands \\ Correspondence should be addressed to Linas Kliucininkas; linas.kliucininkas@ktu.lt
}

Received 24 April 2014; Revised 16 May 2014; Accepted 18 May 2014; Published 10 June 2014

Academic Editor: Prashant Kumar

Copyright (C) 2014 Jonas Matulevicius et al. This is an open access article distributed under the Creative Commons Attribution License, which permits unrestricted use, distribution, and reproduction in any medium, provided the original work is properly cited.

\begin{abstract}
Electrospun polyamide 6 (PA 6) and polyamide 6/6 (PA 6/6) nanofibers were produced in order to investigate their experimental characteristics with the goal of obtaining filtration relevant fiber media. The experimental design model of each PA nanofibers contained the following variables: polymer concentration, ratio of solvents, nanofiber media collection time, tip-to-collector distance, and the deposition voltage. The average diameter of the fibers, their morphology, basis weight, thickness, and resulting media solidity were investigated. Effects of each variable on the essential characteristics of PA 6/6 and PA 6 nanofiber media were studied. The comparative analysis of the obtained PA 6/6 and PA 6 nanofiber characteristics revealed that PA 6/6 had higher potential to be used in filtration applications. Based on the experimental results, the graphical representation-response surfaces-for obtaining nanofiber media with the desirable fiber diameter and basis weight characteristics were derived. Based on the modelling results the nanofiber filter media (mats) were fabricated. Filtration results revealed that nanofiber filter media electrospun from PA6/6 8\% (w/vol) solutions with the smallest fiber diameters $(62-66 \mathrm{~nm}$ ) had the highest filtration efficiency $\left(\mathrm{PA} 6 / 6 \_30=84.9-90.9 \%\right)$ and the highest quality factor $\left(\mathrm{PA} 6 / 6 \_10=0.0486-0.0749 \mathrm{~Pa}^{-1}\right)$.
\end{abstract}

\section{Introduction}

Recent advances in nanofiber material research provide a platform for further innovative biomedical and technical applications [1]. The main techniques for nanofiber based material production are multicomponent fiber spinning, centrifugal spinning, modular meltblowing, pressurised gyration process, and electrospinning $[2,3]$. The latter is the most popular technique due to its simplicity and inexpensive instrumental setup [4-6]. Filtration is one of the most important areas of the electrospun fiber application. Due to the unique characteristics, such as large surface-area-tovolume ratio and low basis weight, nanoporous structures, as well as the uniform size electrospun nanofiber materials, could be employed for a full scale air filtration application [7-9]. Decreasing fiber diameter leads to better filtration efficiency [10]; the ability to capture submicron particles is greater for nanofibers than that of the larger ones. Thus, electrospun nanofibers can have a remarkable impact in HEPA (high efficiency particulate air) and ULPA (ultralow penetration air) filtration $[9,11-13]$.

Electrospun nanofibers from polyamide (PA) already found a number of applications [14-21]. PA has a superior fiber forming ability. It is a biodegradable and biocompatible synthetic polymer with good mechanical properties, which are further enhanced by hydrogen bonds. Unlike other polymers, such as polyethylene oxide and polyvinyl alcohol, PA is resistant to both water and humidity. PA can be dissolved in the solutions of formic or formic/acetic acids; this ensures environment-friendly processes in comparison with other solvents such as dimethylformamide (DMF), which is toxic, according to the EU directive 67/548/EEC. PA 6 and PA $6 / 6$ are the most studied polyamide species, but the discussion of their applications for filtration purposes is rather 


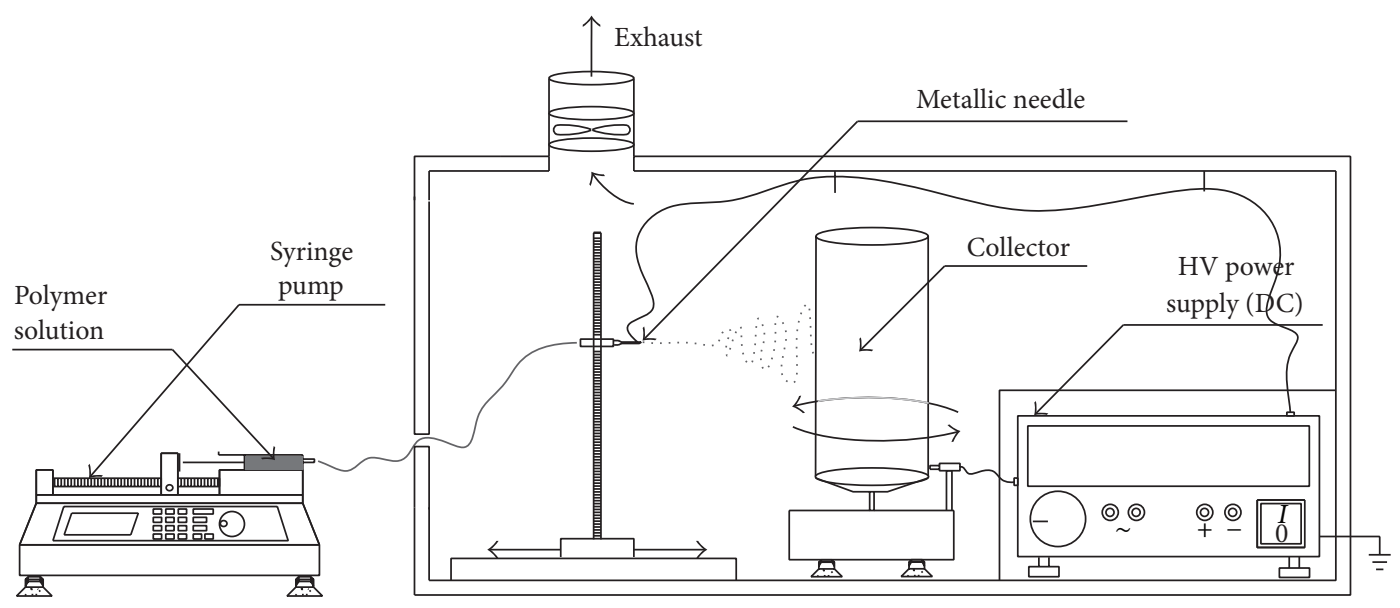

FIGURE 1: Schematic of the used electrospinning system.

limited [11, 22,23]. Recent reports on the properties of electrospun nylon-6 nanofibers showed PA as a particularly attractive material for filtration applications [21].

The aim of this study is the comprehensive design and characterisation of electrospun PA 6 and PA 6/6 nanofiber media suitable for air filtration applications. We have employed principal component analysis modelling to systematically investigate the effects of the fiber deposition parameters, such as polymer solution composition and electrospinning conditions, on the filtration properties of the obtained nanofibers (fiber diameter, basis weight, thickness, and solidity). We derived response surfaces of the nanofiber media that allow determining the set of parameters necessary to obtain specific nanofiber characteristics for a particular filtering application. Finally, based on the modelling results, we selected the best fiber deposition parameters, fabricated nanofiber filter media (mats), and characterised their filtration properties.

\section{Materials and Methods}

2.1. Fabrication of Nanofiber Media. The PA 6 and PA $6 / 6$ pellets, as well as formic acid (85\%), acetic acid (99\%), and dichloromethane (98\%), were purchased from SigmaAldrich. All materials were used without any purification. The homogeneous solutions were obtained by dissolving PA in formic acid (FA), formic acid/acetic acid (FA/AA) $3: 2$ (vol/vol), or formic acid/dichloromethane (FA/DCM) $3: 2$ and $3: 1(\mathrm{vol} / \mathrm{vol})$ via mechanical stirring at room temperature. The PA $6 / 6$ concentrations in the solutions were $8 \%, 11 \%$, and $14 \%$ (w/vol), while PA 6 concentrations were $20 \%, 24 \%$, and $28 \%(\mathrm{w} / \mathrm{vol})$. The concentration ranges chosen for both PA species ensured formation of well-defined nanofibers.

Single-needle system was used in electrospinning experiments (see Figure 1). The electrospinning device consisted of a high voltage DC power supply based on Flyback principle. The grounded electrode was connected to the collector and the high voltage electrode was connected to the needle of the syringe. The PA solutions were loaded into a syringe equipped with a needle, fixed horizontally on the syringe pump (LSP011A, Baoding Longer Precision Pump Co., Ltd., China). Electrospun fibers were collected on a vertically positioned cylindrical collector coated with polyester fabric and rotating at linear speed of $150 \mathrm{~cm} / \mathrm{min}$ (rotation frequency of $6 \mathrm{rpm}$ ). The electrospinning process parameters used for PA $6 / 6$ were as follows: voltage of 12 and $20 \mathrm{kV}$, feed rate of $0.25 \mathrm{~mL} / \mathrm{h}$, and tip-to-collector distance (TTCD) of 6 and $12 \mathrm{~cm}$. The electrospinning process parameters for PA 6 were voltage of 12 and $20 \mathrm{kV}$, feed rate of $0.25 \mathrm{~mL} / \mathrm{h}$, and tip-to-collector distance of 7 and $14 \mathrm{~cm}$. The electrospinning was carried out in an enclosed Plexiglas chamber at ambient conditions $\left(\sim 20^{\circ} \mathrm{C}\right.$ and $\sim 40 \%$ relative humidity). Collected samples of the electrospun nanofibers were dried in vacuum at room temperature for several hours.

2.2. Characterization of Electrospun Fiber Media. The morphology of the fiber networks and the corresponding diameters of the fibers were investigated using scanning electron microscope (SEM) and atomic force microscope (AFM). Scanning electron microscopy images were acquired using Hitachi S-4800 microscope equipped with a cold field emitter operating at $2 \mathrm{kV}$ accelerating voltage. No specific sample preparation was performed before imaging. Atomic force microscopy was performed using Asylum Research MFP-3D instrument in alternating current (AC) mode. Mikromasch NSC-15 silicon cantilevers with $\mathrm{Al}$ coated backside, typical resonant frequency of $325 \mathrm{kHz}$, and force constant of $40 \mathrm{~N} / \mathrm{m}$ were used in all experiments. The samples for AFM analysis were electrospun on $\mathrm{Si}$ wafer surface attached to the collector.

The basis weight of the nanofibers was estimated by dividing the weight of the sample mat by its effective area. The effective sample area for the basis weight estimation was taken from within $10 \mathrm{~mm}$ radius from the center of the mat sample and was measured using a microbalance (MXA-5, Radwag, Poland). The thickness of the nanofiber filter mat was measured in the center of the nanofiber media using a digital micrometer with a measurement resolution of $1 \mu \mathrm{m}$. The average diameter of the fibers was determined 


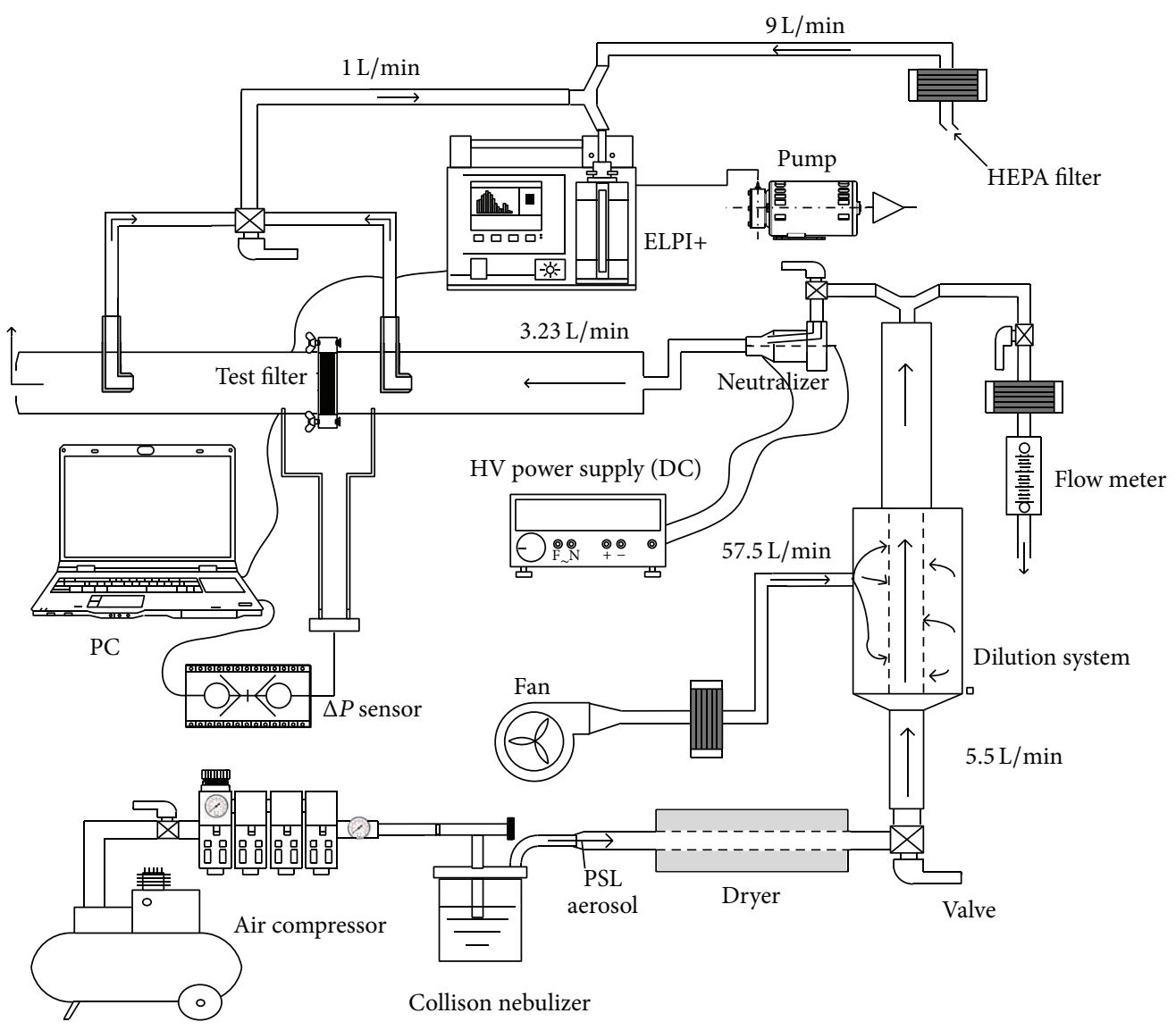

FIGURE 2: Experimental setup for testing filtration efficiency and pressure drop.

by analyzing SEM images with ImageJ (NIH, USA) image analysis software. For each sample fiber diameters were measured at 50 different points.

Solidity, which is presented in Table 1, is often referred to as packing density. It shows the volume of solids in the medium per unit volume of medium. Solidity is the opposite value of porosity $(\varepsilon=1-\alpha)$, which is the three-dimensional volume void fraction of the medium [24]. The solidity of the nanofibers was estimated using the following empirical formula [7]:

$$
\alpha=\frac{W}{\rho \cdot Z}
$$

where $W$ is the basis weight of the nanofiber filter (mass of the nanofibers per unit filter area), $\rho$ is the density of the fiber material, and $Z$ is the thickness of the nanofiber filter mat.

The mathematical design of the experiments was based on a D-optimal-interaction model developed within MODDE 7 software (Umetrics AB, Sweden). Each experiment for PA 6 and PA 6/6 was conducted with the predefined combination of variables that included the polymer concentration, the nature of the solvent, the deposition voltage, the tip-tocollector distance, and the collection time. Once all the responses (fiber diameter, basis weight, thickness, and solidity) of the experiments were obtained, the partial least squares (PLS) method was applied to develop a polynomial model relating the factors to the responses. PLS deals with all factors simultaneously taking their covariances into account. The advantage of this model is a reliable interpretation and prediction of the interaction between experimental factors and responses.

The electrospinning of PA fibers was carried out via 34 controlled experiments as recommended by D-optimalinteraction model, 17 experiments for each grade of PA. Seven different combinations of polymer concentrations and solvents were applied for each PA species. Two separate models, one for the PA $6 / 6$ and one for the PA 6 , were developed. Goodness of fit $\left(R^{2}\right)$ and goodness of prediction $\left(Q^{2}\right)$ parameters were estimated as measures for the accuracy of the model.

2.3. Filtration Performance of Nanofiber Media. The experimental setup shown in Figure 2 was used to measure filtration efficiency and pressure drop. The dry and clean air after the filter system was provided to the Collison nebulizer (Model CN 24 J, BGI Inc., MA, USA). In order to generate the monodisperse PSL particles (diameter $=100$ and $300 \mathrm{~nm}$, purchased from Sigma-Aldrich) the Collison nebulizer was filled with polystyrene latex (PSL) solution suspended in deionized water (DI) $(0.2 \mathrm{~mL}$ of PSL suspension in $100 \mathrm{~mL}$ DI). The aerosol particles released from the nebulizer were dried in the diffusion dryer packed with silica gel and were 


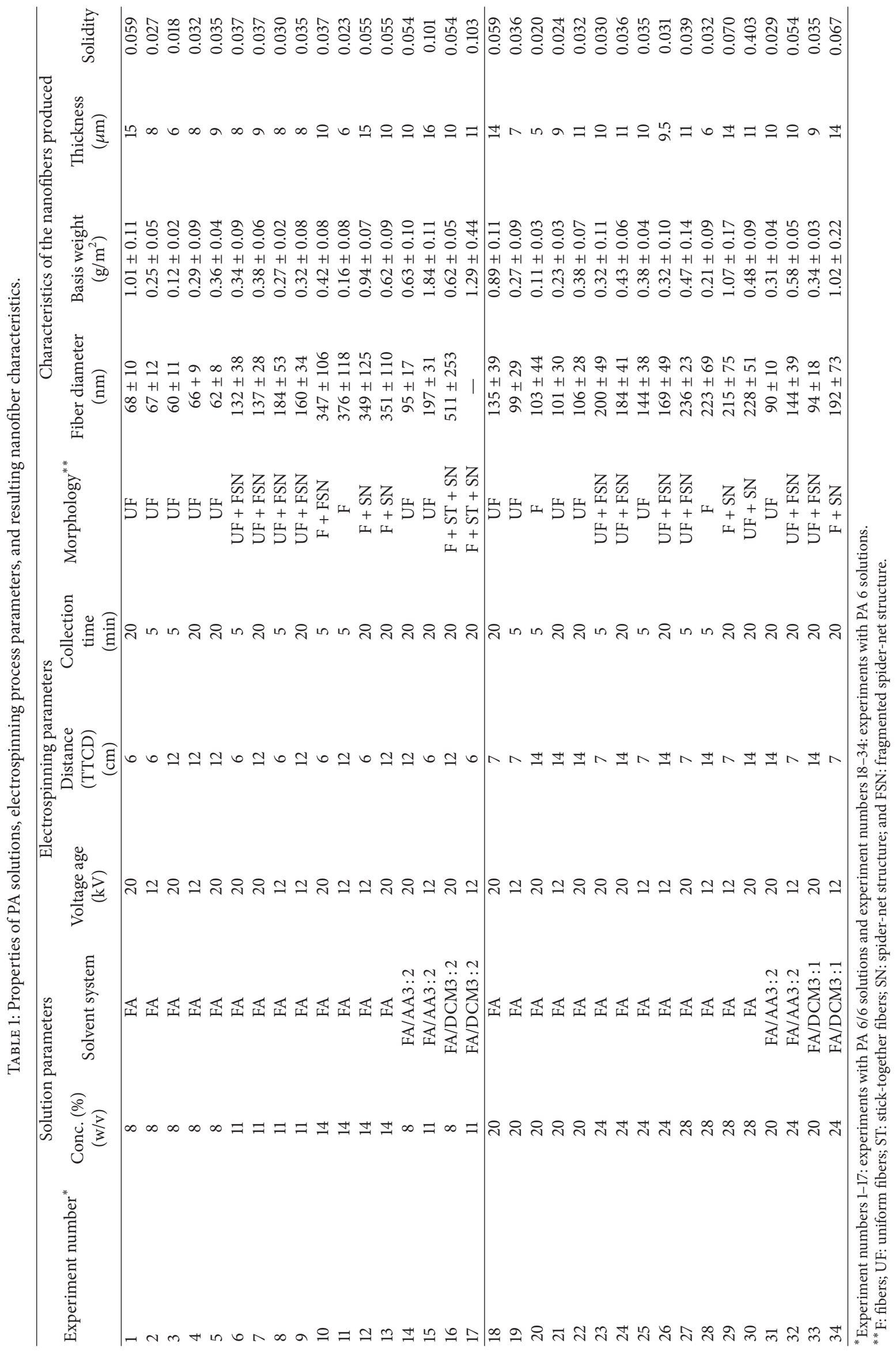


supplied to the dilution chamber. After passing the dilution chamber, the aerosol flow was split into two paths. In the first subflow aerosol particles passed the neutralizer (DC corona discharge) [25] and were directed to the elongated tube with the filter inside. The flow rate of $3.23 \mathrm{~L} / \mathrm{min}$, which corresponds to the face velocity of $5.3 \mathrm{~cm} / \mathrm{s}$ for $36 \mathrm{~mm}$ diameter filter, was applied. The second subflow after HEPA filter was used to control the flow rate of the entire filtration system. The pressure drop was registered by the pressure sensor (Model P300-5-in-D, Inc., Mooresville, USA). The concentrations of aerosol particles before and after the tested filter media were measured by ELPI+ instrument (Dekati Ltd., Finland) connected to the computer.

Before starting filtration tests all the filters were neutralized by charger neutralizer; thus, electrostatic effect was avoided. The evaluation of nanofiber media filtration properties was performed only for selected and additionally fabricated PA media with longer electrospinning duration.

Nanofiber filters having high filtration efficiency at a low pressure drop are the most desirable in filtration. Both aspects are considered into quality factor $(\mathrm{QF})$ of the filter, also referred to as figure of merit of the filter, which is often used to evaluate the filtration performance of filters. The quality factor is defined as [10]

$$
\mathrm{QF}=-\frac{\ln (1-E)}{\Delta P},
$$

where $E$ is filtration efficiency and $\Delta P$ is the pressure drop across the filter.

\section{Results and Discussion}

3.1. The Characteristics of the Electrospun $P A$ and $P A 6 / 6$ Nanofibers. Morphology and fiber diameter of the electrospun fibers were obtained from SEM images shown in Figure 3, whereas basis weight and thickness were derived from experimental measurements, while solidity values were estimated using formula (1). Composition of the initial PA solutions, the electrospinning process parameters, and the resulting nanofiber media characteristics, relevant to air filtration, are presented in Table 1. The fibers of PA 66 were electrospun from polymer concentration ranges from 8 to $14 \%$ (w/vol), while fibers of PA 6 were electrospun-at the concentrations ranges from 20 to $28 \%$ (w/vol). The compositional concentration of the precursor solutions appeared to be the main parameter influencing the fiber diameter, as discussed in Section 3.2. The selected ranges of the concentrations resulted in a varying fiber diameter with the corresponding values ranging from 60 to $376 \mathrm{~nm}$ for PA $6 / 6$ and from 99 to $236 \mathrm{~nm}$ for PA 6. PA $6 / 6$ revealed a wider electrospinnable range in comparison to PA 6. This agrees with the previously reported data on the electrospinning of PA 6/6 [14] and on PA 6 [17].

The values of the calculated basis weight were in the range from 0.12 to $1.84 \mathrm{~g} / \mathrm{m}^{2}$ for PA $6 / 6$ and from 0.11 to $1.07 \mathrm{~g} / \mathrm{m}^{2}$ for PA 6, while the thickness values were from 6 to $16 \mu \mathrm{m}$ for PA 6/6 and 5-14 $\mu \mathrm{m}$ for PA 6. An apparent dependence of the basis weight and thickness on the collection time can be inferred. However, as discussed in Section 3.2, the collection time was not the only factor affecting the above motioned nanofiber mat parameters. The solidity increased simultaneously with the basis weight in most cases but the increase rate dropped after the basis weight value of $0.6 \mathrm{~g} / \mathrm{m}^{2}$ was reached (experiments numbers 15 and 17 were not considered).

The electrospinning technique can form various nanofiber structures depending on the solvent system and the polymer concentration. The representative SEM images of PA 6/6 and PA 6 fibers are shown in Figure 3. Based on the structural differences, electrospun PA mats were grouped into three basic types of morphologies: fibers (F), uniform fibers (UF), and stick-together fibers (ST). The morphology of $\mathrm{F}$ was characterized by the wider distribution of fiber diameter within the electrospun mat (standard deviation of fiber diameter $(\mathrm{SD}) /$ fiber diameter $(\mathrm{FD})>0.3$ ), while UF morphology of the electrospun mat can be characterized as smooth with a narrower distribution of the fiber diameters $(\mathrm{SD} / \mathrm{FD}<0.3)$. Due to the residual solvent deposited on the collector, the structure of the coalesced fibers can be observed in ST morphology samples. In most cases, UF morphology was observed for lower to middle range of polymer concentrations, for example, 8 and $11 \% \mathrm{w} / \mathrm{vol}$ for PA $6 / 6$ and 20 and 24\% w/vol for PA 6 . Only in case of PA 6 with polymer concentration of $28 \%$ w/vol UF morphology was observed (experiments numbers 27 and 30 in Table 1). It is worth noting that FA/AA solvent mixture in ratio of $3: 2 \mathrm{vol} / \mathrm{vol}$ for both grades of polyamides contributed to especially smooth and uniform fibers (Figures 3(e) and 3(f)).

The detailed analysis of the SEM images revealed the presence of the nanoscale fibers in the range of few tens of nanometers, known as spider-net like (SN) fibers (Figures $3(\mathrm{c}), 3(\mathrm{~d})$, and $3(\mathrm{~g}))[21,26]$. The SN structures have been shown to yield improved mechanical properties of PA mats [26]. The formation of the SN structure may be explained by the formation of the hydrogen bond between the main polyamide chain of the amide groups $(\mathrm{CO}-\mathrm{NH})$ and oligomeric, monomeric ionic species $\left(-\mathrm{CONH}_{2}-^{+}\right)$[21]. The presence of the oligomeric and monomeric ions in solution is due to the formic acid, which is capable of attacking the lactam to produce a series of short oligomers and monomers [21]. We propose that these additional SN structures can be advantageous for air filtration applications due to the formation of a denser structure of filtering layer, thus increasing filtration efficiency due to the particle interception mechanism. Thus, formation of the spider-net like morphology is desirable in case of the application of nanofiber layer for filtration.

The SN structures were observed in electrospun nanofibers obtained from both polyamides. However, not all experiments were successful in yielding $\mathrm{SN}$ morphology (see Table 1). It can be seen that the polymer concentration was one of the main factors influencing formation of the $\mathrm{SN}$ structures. These structures were observed for higher polymer concentrations of both polyamides, for example, $14 \%$ $\mathrm{w} / \mathrm{vol}$ for PA $6 / 6$ and $28 \% \mathrm{w} / \mathrm{vol}$ for PA6 solutions in formic and formic/acetic acids (see experiments numbers 12, 13, 29, 


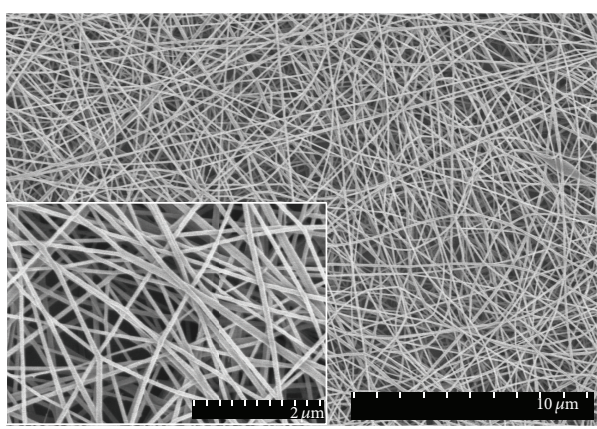

(a)

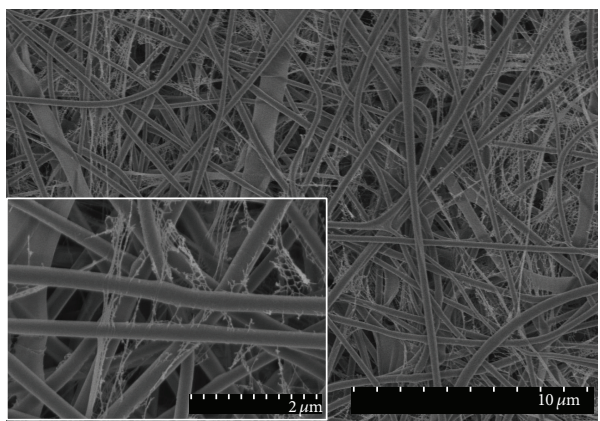

(c)

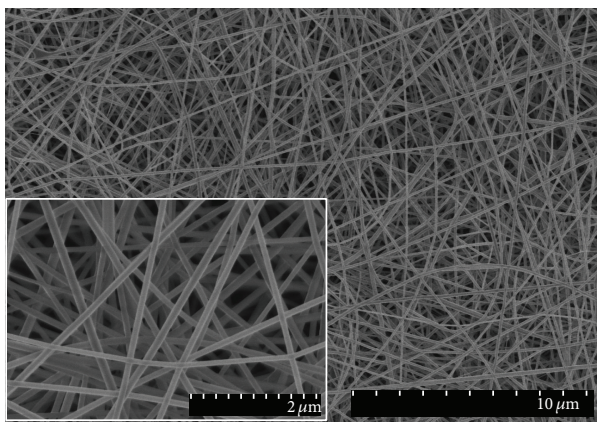

(e)

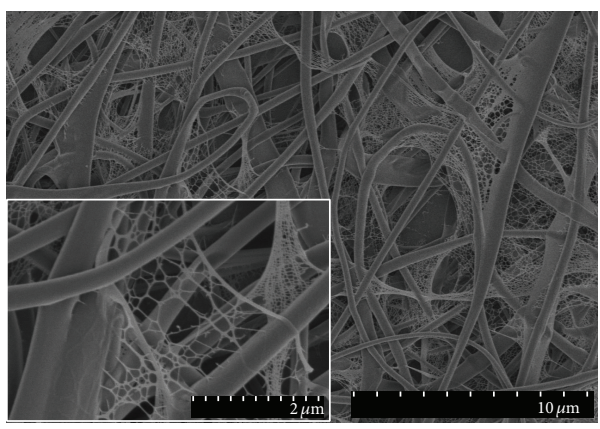

(g)

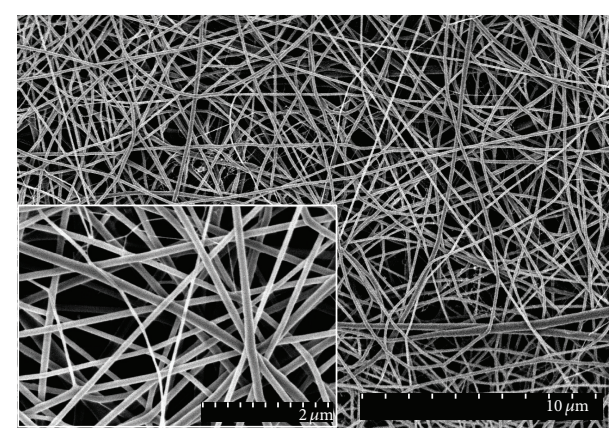

(b)

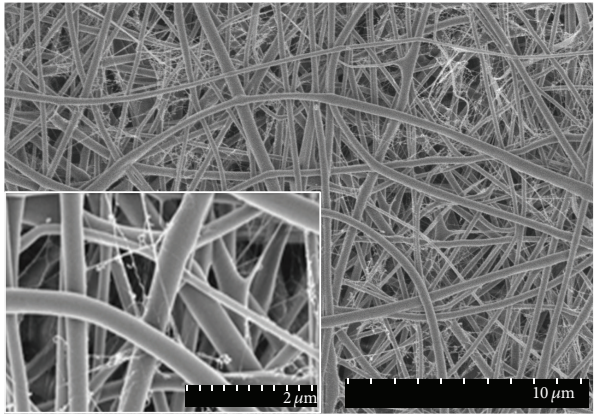

(d)

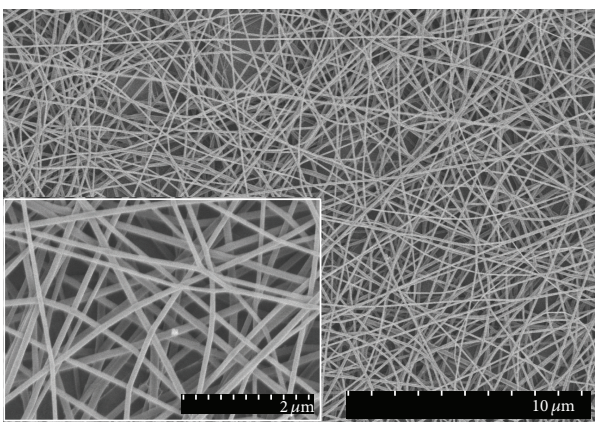

(f)

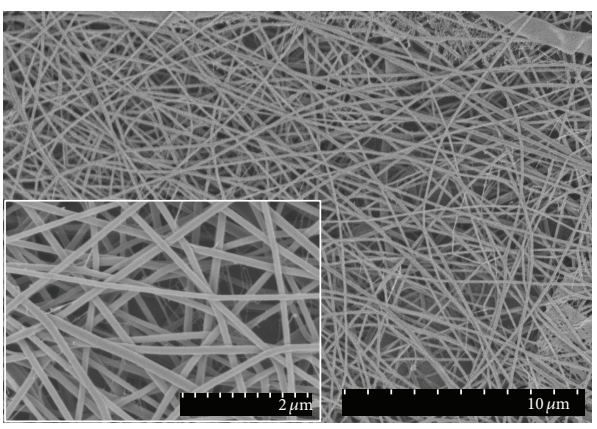

(h)

Figure 3: SEM images of the PA nanofibers deposited using (a) PA 6/6 8\% w/vol in FA, (b) PA6 20\% w/vol in FA, (c) PA 6/6 14\% w/vol in FA, (d) PA 6 28\% w/vol in FA, (e) PA 6/6 8\% w/vol in FA/AA $3: 2 \mathrm{vol} / \mathrm{vol}$, (f) PA 6 20\% in FA/AA 3:2 vol/vol, (g) PA 6/6 8\% w/vol in FA/DCM $3: 2 \mathrm{vol} / \mathrm{vol}$, and(h) PA $620 \% \mathrm{w} / \mathrm{w}$ in FA/DCM $3: 1 \mathrm{vol} / \mathrm{vol}$.

and 30). In the case of PA solutions in formic acid/DCM as a solvent, $\mathrm{SN}$ or fragmented spider-net (FSN) structures for lower and middle ranges of concentrations were observed (see experiments numbers 16, 17, 33, and 34). The formation of SN structure in experiment number 16 can be explained by the increased flow of PA solution. Prolonged utilization of the solvent system containing volatile compounds, such as DCM with the boiling point of $39^{\circ} \mathrm{C}$, causes clogging of the nozzle during the electrospinning process, especially in low humidity environment [1]. The clogging of the nozzle 

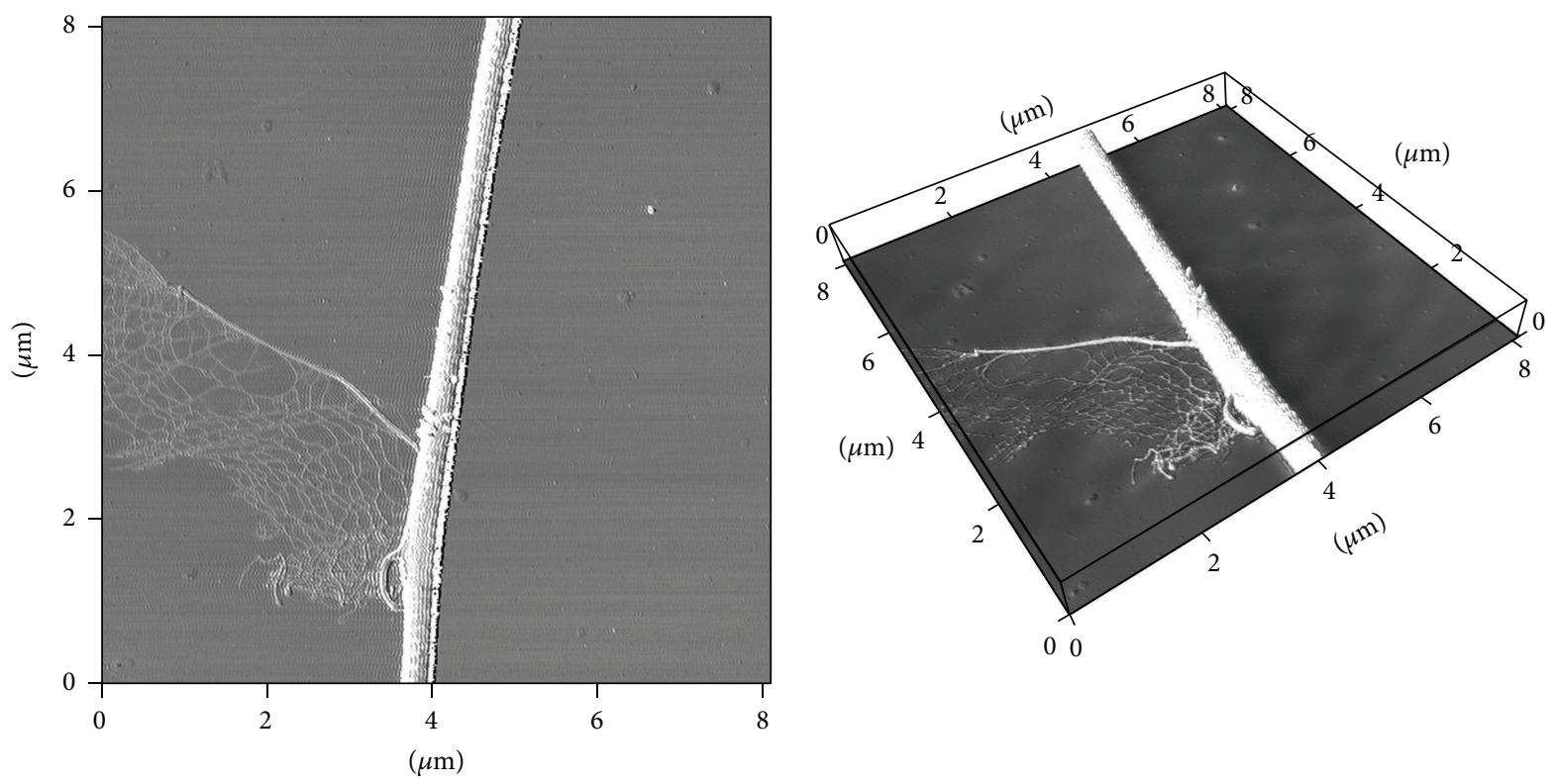

FIgURE 4: Two-dimensional and three-dimensional AFM images of a single polyamide fiber and the spider-net like (SN) structure.

was also observed in our study. To avoid clogging, feed rates of the solutions with DCM were increased twofold, which in turn produced moister nanofibers. Moister nanofibers of electrospun mats experiments numbers 16 and 17 were thicker and were characterized by the stick-together morphology. The effective distance between the short oligomeric and monomeric ionic species and amide groups was shorter in such morphology. Therefore, the probability of the hydrogen bond formation mechanism was more viable. Another parameter influencing formation of the $\mathrm{SN}$ structure was the collection time of the nanofiber mat. Only nanofiber mats with 20 min collection time demonstrated the SN structure (experiments numbers 12, 13, 16, 17, 29, 30, and 34).

The increased collection time and polymer concentration of PA as well as the presence of DCM were the main factors influencing the formation of SN structure, while lower voltage $(12 \mathrm{kV})$ and shorter collection time (5 min) were factors having no effect on the formation of SN structures (experiments numbers. 11, 25, and 28). Our findings agree with the results reported previously [21] and could be explained by the fact that lower voltages invoke less ionization of the polyamide solution, resulting in absence of the SN structures. It was demonstrated that formation of the multilayer SN structures could be increased by using inorganic salts $\left(\mathrm{NaCl}, \mathrm{KBr}\right.$, and $\left.\mathrm{CaCl}_{2}\right)$ for PA 6 and polyvinyl alcohol (PVA) electrospun nanofiber mats [26].

An AFM analysis allowed for a more detailed analysis of the formed SN structure. Figure 4 shows single fiber and the attached SN structure of PA 6/6 (experiment number 16). The width and the height of a single fiber were $465 \mathrm{~nm}$ and $220 \mathrm{~nm}$, while width and height measurements of SN fibers ranged between 9-28 $\mathrm{nm}$ and 7-15 $\mathrm{nm}$, respectively.

3.2. PLS Analysis of the Deposition Parameters on the Resulting Nanofiber Characteristics. The data collected during the experimental runs were fitted to the PLS model with the aim of establishing a relationship between the factors (voltage, tip-to-collector distance, collecting time, solvent system, and polymer concentration) and their responses (fiber diameter, basis weight, and thickness) of the obtained nanospun fibers. The solidity was not included in the modeling since it is a function of the fiber basis weight and the thickness of the fiber media (formula (1)). Calculated values of the solidity for the samples obtained in this work are presented in Table 1. The developed models were rather accurate, as indicated by their high values of $R^{2}$ (higher than 0.90 for all filtering parameters), while the $Q^{2}$ values ranged from 0.62 to 0.71 .

The results of the effects of modelling of polymer solution and electrospinning parameters on PA 6 and PA 6/6 nanofiber media characteristics are presented in Figure 5. The positive values stand for factors positively affecting the responses, while negative values represent factors reducing the values of the responses. The error bars represent confidence intervals indicating the uncertainty of each factor. The factors were considered statistically insignificant if their confidence intervals included zero.

The analysis of the experimental results agrees with the previous data $[16,18,27]$ where the polymer concentration had a substantial impact on the fiber diameter (see Figures 5(a) and 5(d)). Lower polymer concentrations influenced greater mobility of the polymer chains and larger instabilities of polymer jet during the electrospinning. This induced greater stretching of the polymer jet resulting in the lower nanofiber diameters. Higher polymer concentrations caused a restricted movement of the polymer chain and the jet of polymer was stabilized [17].

Another factor having great influence on the fiber diameter was the presence of DCM in solvent systems (Figure 5(a)). For PA 6/6, a rapid evaporation of DCM from FA/DCM $3: 2 \mathrm{vol} / \mathrm{vol}$ system (Exp. no. 16 and 17) obstructed continuous 


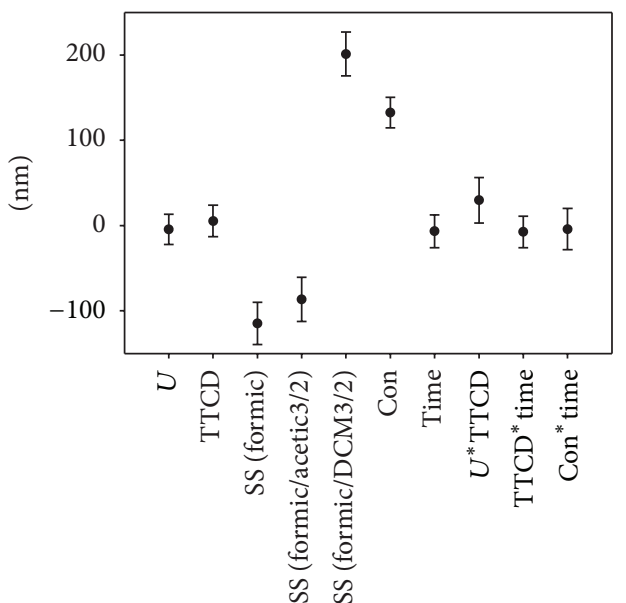

(a)

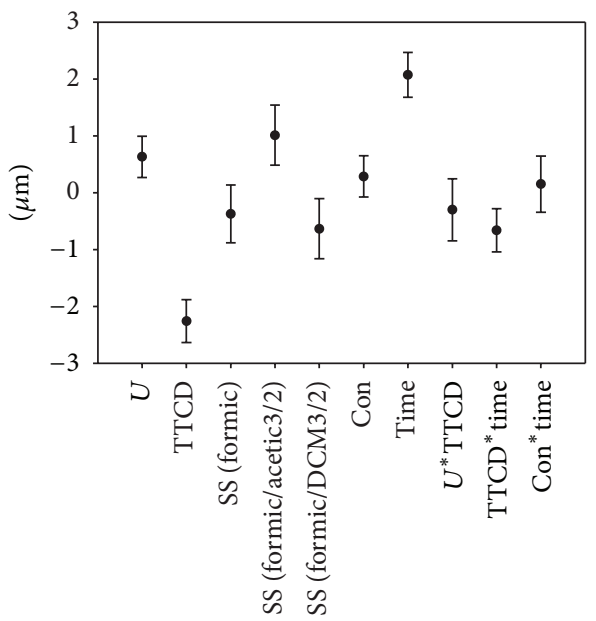

(c)

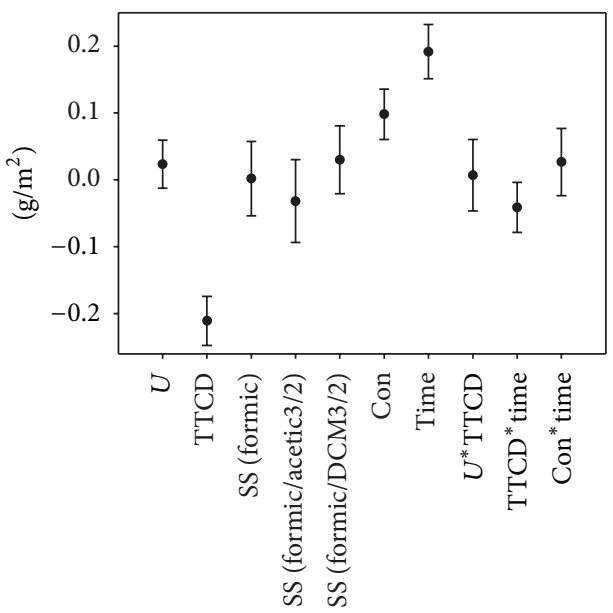

(e)

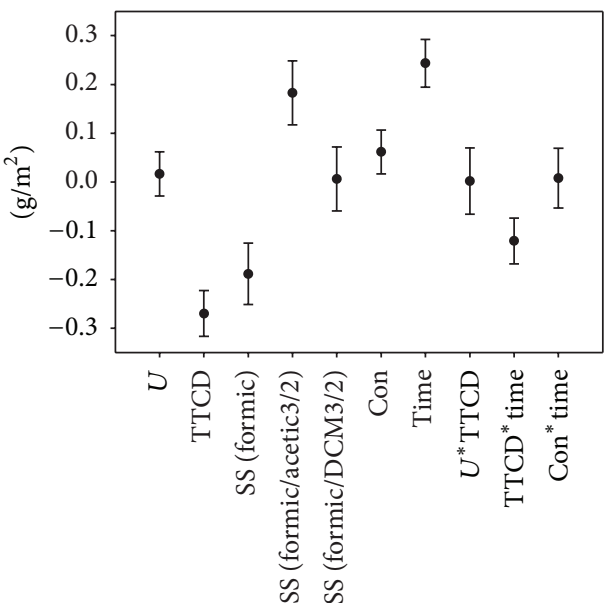

(b)

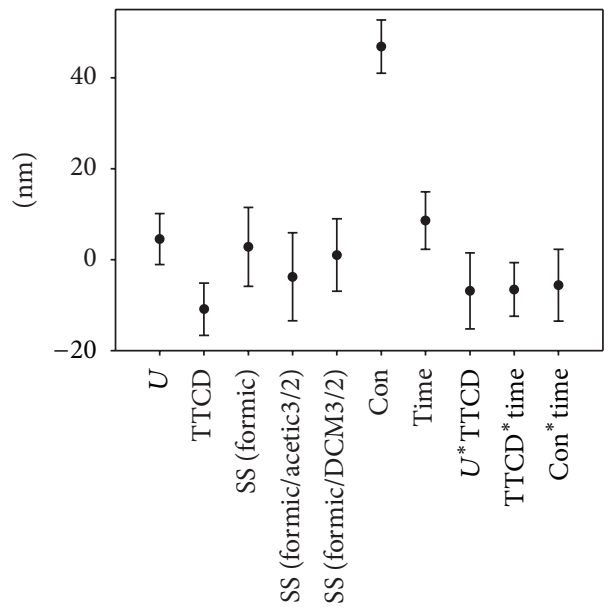

(d)

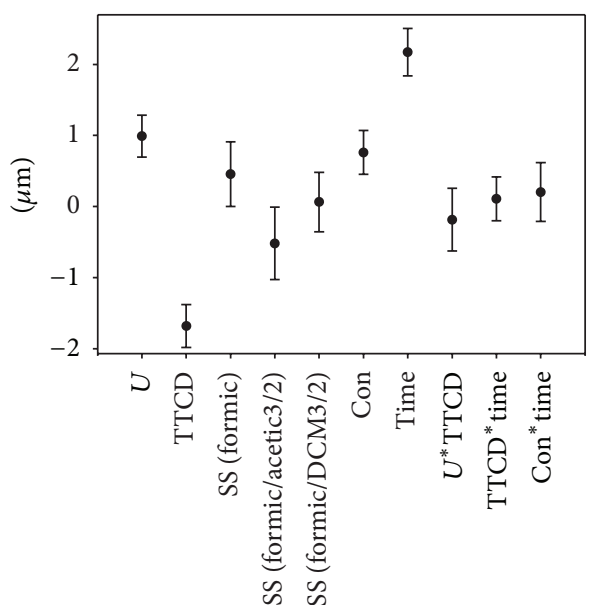

(f)

FIGURE 5: The inputs of polymer solution and electrospinning parameters on air filtration essential nanofiber media characteristics: (a) PA 6/6 fiber diameter, (b) PA 6/6 basis weight, (c) PA 6/6 thickness, (d) PA 6 fiber diameter, (e) PA 6 basis weight, and (f) PA 6 thickness. $U$-voltage, TTCD—-tip-to-collector distance, SS—-solvent system, and Con-concentration. 
flow of the polymer solution to the nozzle. After the increase of the feed rate of FA/DCM 3:2 vol/vol, the fiber diameter increased. However, a stick-together morphology became noticeable. In contrast, when PA 6 nanofibers were electrospun with FA/DCM $3: 1 \mathrm{vol} / \mathrm{vol}$ solvent system (experiments numbers 33 and 34), influence of DCM solvent on the fiber diameter was found to be negligible. FA/AA 3:2 (vol/vol) solvent system demonstrated an inverse effect on the fiber diameter (see Figures 5(a) and 5(d)).

The effects of TTCD and deposition voltage on the resulting nanofiber diameter were analyzed for each concentration of the polymer solution using experimental data shown in Table 1. For PA 6/6, the TTCD had no significant effect on the fiber diameter, whereas an increase in the voltage resulted in a decrease of the fiber diameter. Contrary to PA 6, a higher voltage $(20 \mathrm{kV})$ combined with a shorter TTCD $(7 \mathrm{~cm})$ resulted in the increase of PA 6 fiber diameter. A stronger electric field $(\mathrm{kV} / \mathrm{cm})$ was responsible for the formation of thicker electrospun PA 6 fibers, whereas the smallest fibers were obtained at medium values of the electric field. In case of the higher voltage, the jet induced by higher electrostatic forces and higher repulsive forces favored deposition of smaller fibers [17]. On the other hand, the higher voltage may cause a higher mass flow resulting in thinner fibers. A shorter TTCD caused a deposition of a moister jet and resulted in the coalescence of the fibers leading to a thicker fiber mat, while the longer TTCD enhanced the evaporation of solvent leading to thinner fibers. Similar findings were also reported previously [17].

The effects of TTCD and the collection time on the basis weight and the thickness of nanofibers are presented in Figures 5(b) and 5(e) and Figures 5(c) and 5(f). TTCD had a slightly larger effect on the basis weight and thickness of PA 6/6 fibers with the corresponding negative responses of $-0.27 \mathrm{~g} / \mathrm{m}^{2}$ and $-2.26 \mu \mathrm{m}$, respectively; collection time had a positive modeled response on the basis weight of PA 6/6 fibers with the corresponding value of $0.24 \mathrm{~g} / \mathrm{m}^{2}$, while collection time had also a positive modeled response on the thickness of PA 6 fibers with the value of $2.17 \mu \mathrm{m}$. Modeling results clearly showed that basis weight and thickness of fibers were dependent not only on the collection time, but also on TTCD.

\subsection{Optimization of the Polymer Solution and Electrospinning} Parameters. The optimization of the polymer solution and electrospinning parameters provides prerequisites for practical usage of the nanofibers for larger scale air filtration applications. Based on the results of complex experimental design data we derived response surface plots for obtaining nanofiber media with desirable fiber characteristics (see Figure 6).

The PLS analysis showed that the fiber diameters of both PA materials were influenced the most by a single factora precursor concentration, while other factors were almost negligible. However, voltage was also chosen as an important factor for the prediction of nanofiber characteristics, as discussed in Section 3.2.

In order to form nanofibers with the specific diameter (see Figure 6(a) for PA 6/6 and Figure 6(b) for PA 6) the determined values of the polymer concentration and electrospinning voltage may be obtained from the data shown in Figure 6. At the same time, other parameters (tip-to-collector distance-9 $\mathrm{cm}$ (for PA 6/6) and $10.5 \mathrm{~cm}$ (for PA 6), solvent system-FA, and collection time-12.5 min) should be maintained constant. Additionally, deposition parameterscollection time and the TTCD-of fibers with the specific basis weight (see Figure 6(c) for PA 6/6 and Figure 6(d) for PA 6) may be also determined from Figure 6, keeping other parameters (voltage- $16 \mathrm{kV}$; concentration-11\% (for PA 6/6) and 24\% (for PA 6); and solvent system-FA) constant.

Finally, the size of particles being filtered is another important parameter in the development of filtration media. It was proposed that for larger particles (100 $\mathrm{nm}$ and above) nanofiber filters with smaller fiber size (150 $\mathrm{nm}$ and below), larger solidity (approximately 0.1 ), and basis weight could be considered [28]. For smaller particles (100 nm and below), filters with larger fiber size (500 $\mathrm{nm}$ and above), smaller solidity (approximately 0.01), and basis weight are suggested [28]. The obtained modeling results (see Figure 6) could be used for optimizing nanofiber fabrication with desirable characteristics, suitable for filtration of different size ranges of particles.

3.4. Filtration Properties. Based on the optimized results, described in Section 3.3, authors fabricated nanofiber filter media samples and tested them in the experimental filtration setup described in Section 2.3. The filtration efficiency, pressure drop, and quality factors for seven PA nanofiber filter media samples were measured (see Table 2). The $300 \mathrm{~nm}$ and $100 \mathrm{~nm}$ aerosol particles were generated by atomising suspensions of polystyrene latex spheres (PSL). The diffusion and the interception mechanisms are comparatively inefficient near the $300 \mathrm{~nm}$ particle size, wherefore $300 \mathrm{~nm}$ particles are characterized as the most penetrating particle size (MPPS) in HEPA filters (European Standard EN1822-1).

For efficient filtration of $300 \mathrm{~nm}$ PSL particles authors referred to experiment number 5 (see Table 1 ) and tested three samples of PA 6/6 nanofiber filter media. The fiber media collection time was defined 10, 20, and $30 \mathrm{~min}$. For efficient filtration of $100 \mathrm{~nm}$ PSL particles authors referred to experiment number 11 (see Table 1) (fiber collection time 10 min.). When testing PA6 nanofiber filter media authors referred to experiment number 30 (see Table 1). The fiber media collection time was defined 10, 20, and $30 \mathrm{~min}$.

The highest filtration efficiency (90.9\%) of $300 \mathrm{~nm}$ particles was received for PA6/6_30 nanofiber filter media, while PA6_30 nanofiber filter media showed the highest filtration efficiency $(87.7 \%)$ of $100 \mathrm{~nm}$ particles. Interestingly, the PA6/6 nanofiber filters with the lowest fiber diameters $(62-66 \mathrm{~nm})$ indicated higher filtration efficiency of $300 \mathrm{~nm}$ particles; however $100 \mathrm{~nm}$ particles were filtered with lower efficiency. This supports hypothesis elaborated in Section 3.3 that nanofiber media with low fiber diameter are more suitable for filtration of bigger particles $(\sim 300 \mathrm{~nm})$, while filter media with comparatively high fiber diameter are more suitable for filtration applications of smaller particles $(\sim 100 \mathrm{~nm})$. Longer fiber collection time makes the fibre 


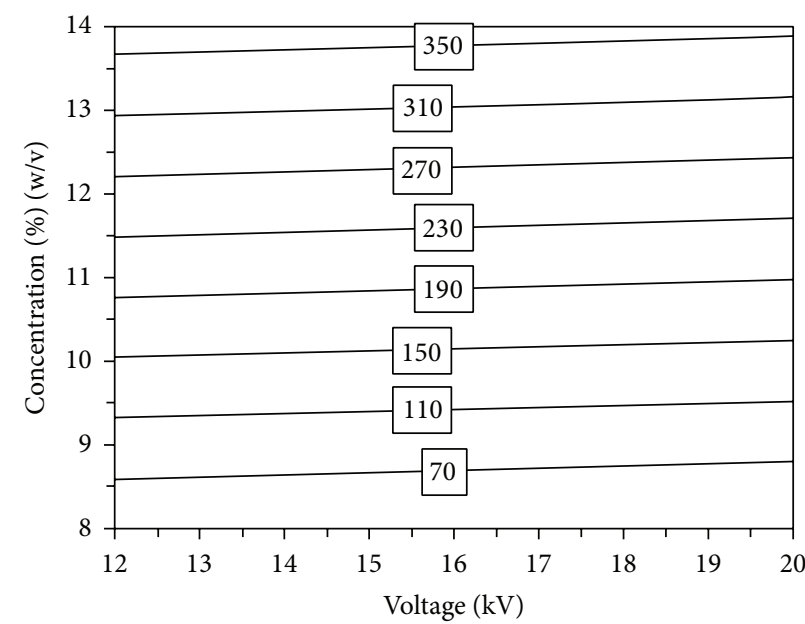

(a)

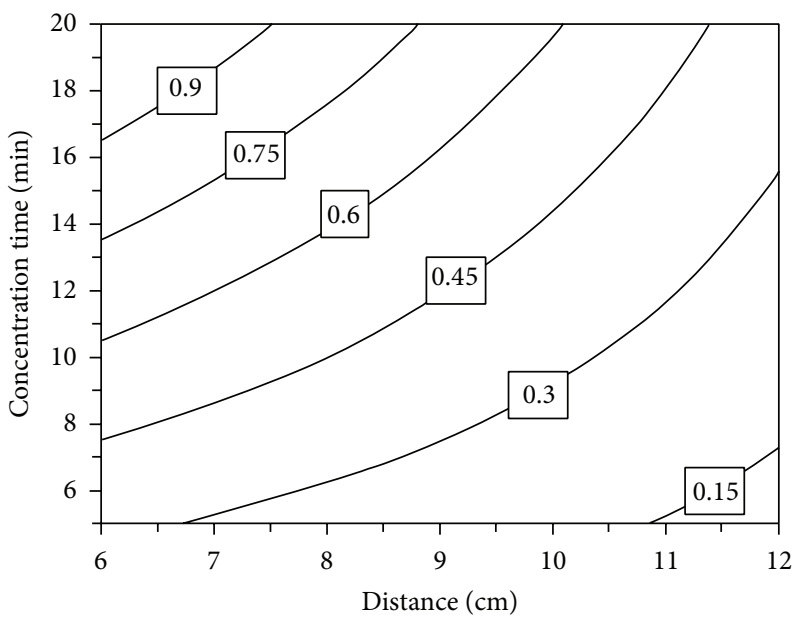

(c)

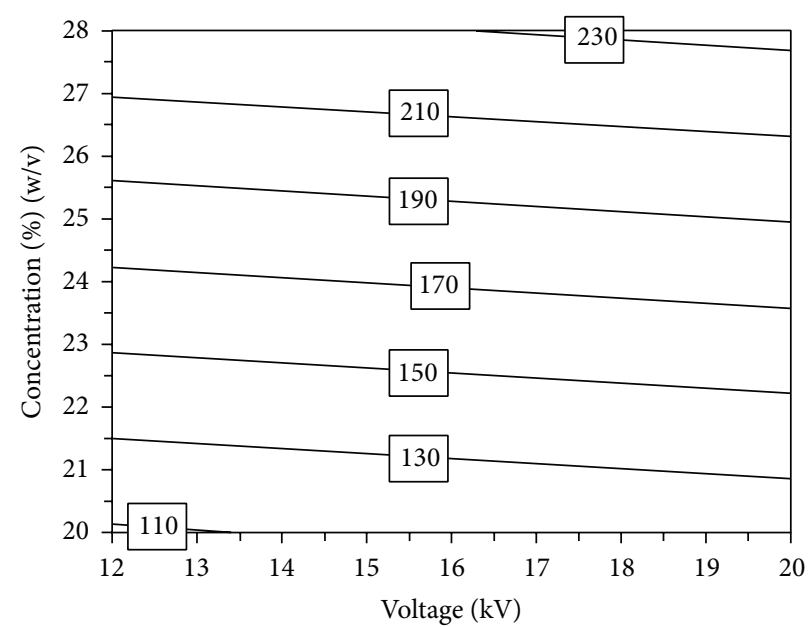

(b)

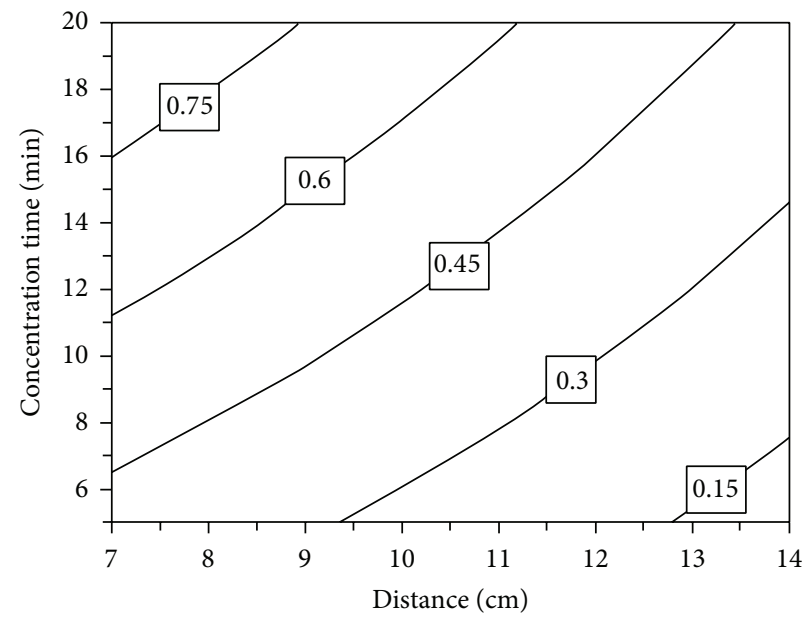

(d)

FIGURE 6: Response surface plots for the prediction of fiber diameter and basis weight through the optimization of the polymer solution and electrospinning parameters: (a) PA 6/6 fiber diameter (TTCD-9 cm; solvent system-FA; and collection time -12.5 min), (b) PA 6 fiber diameter (TTCD-10.5 cm; solvent system-FA; and collection time-12.5 min), (c) PA 6/6 basis weight (voltage-16 kV; concentration-11\%; and solvent system-FA), and (d) PA 6 basis weight (voltage-16 kV; concentration-24\%; and solvent system-FA).

media (mat) denser and has positive effect on filtration efficiency. The obtained results indicate that fibre diameter combined with basis weight of nanofiber media could ensure good filtration efficiency for the specific size of particles.

The measurements of pressure drop for nanofiber filters showed relatively high values; despite this calculated quality factors were comparable to findings of other authors. The overview of filter quality factors obtained by different authors for most penetrating particle size $(300 \mathrm{~nm})$ is presented in Table 3. The results obtained in our study revealed that nanofiber filter media electrospun from PA6/6 8\% (w/vol) solution had high quality factor $\left(0.075 \mathrm{~Pa}^{-1}\right)$.

\section{Conclusions}

Electrospun polymer fibers were deposited via electrospinning with the goal of obtaining controllable mat parameters suitable for air filtration purposes. The concentration of the polyamide 6 and polyamide $6 / 6$ solutions and the composition of the solvent were two major factors affecting the diameter and the morphology of the electrospun nanofibers. Our data show that polyamide $6 / 6$ has a wider electrospinnable range resulting in a wider distribution of the fiber diameter $(60-511 \mathrm{~nm})$ compared to polyamide $6(90-236 \mathrm{~nm})$. Uniform diameter fibers were produced from formic acid and formic acid/acetic acid $3: 2(\mathrm{vol} / \mathrm{vol})$ solvents with lower polymer concentrations, while the collection time combined with the higher concentration of formic acid and formic acid/dichloromethane $3: 2$ and $3: 1(\mathrm{vol} / \mathrm{vol})$ solvents indicated formation of fragmented and continuous spider-net like structures. The stronger electric field was responsible for the formation of thicker polyamide 6 electrospun fibers, whereas the smallest polyamide 6 fibers were obtained at medium values of the electric field. Higher voltage resulted in thicker fibers for polyamide $6 / 6$. The basis weight and the thickness 


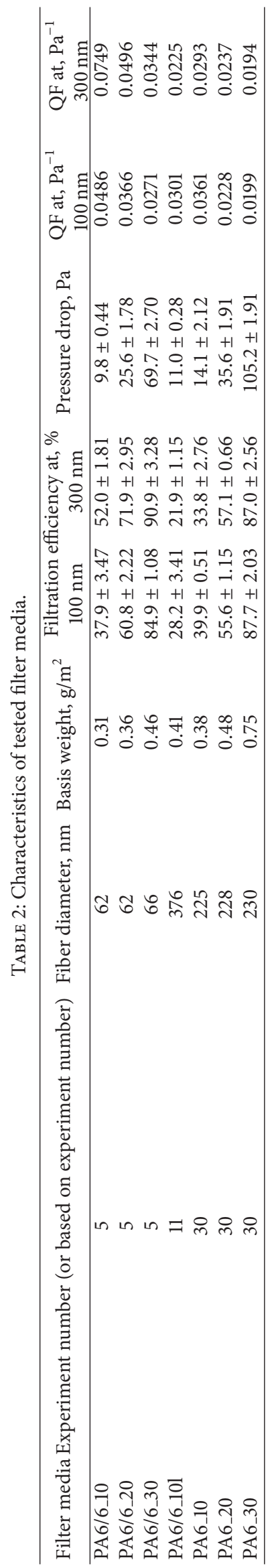


TABLE 3: The overview of filter quality factors obtained by different authors for most penetrating particle size (300 nm).

\begin{tabular}{|c|c|c|c|c|}
\hline Study & Material & $\begin{array}{c}\text { Fiber } \\
\text { diameter, } \mathrm{nm}\end{array}$ & Quality factor, $\mathrm{Pa}^{-1}$ & Comments \\
\hline Wang et al., 2008 [13] & N/A & 150 & $\begin{array}{l}A=0.048 \\
B=0.025 \\
C=0.029 \\
D=0.020\end{array}$ & $\begin{array}{l}\text { Quality factor (QF) for face velocity }(U)=5 \mathrm{~cm} / \mathrm{s} \\
\text { was interpolated }\end{array}$ \\
\hline Zhang et al., 2009 [23] & PA 6 & $\begin{array}{c}110-150 \\
70-100 \\
60-90\end{array}$ & $\begin{array}{l}15 \mathrm{wt} \%=0.036 \\
12 \mathrm{wt} \%=0.020 \\
10 \mathrm{wt} \%=0.016\end{array}$ & $\begin{array}{l}U \text { which was applied in filtration is not known, only } \\
\text { pressure drop for } U=5 \mathrm{~cm} / \mathrm{s} \text { was interpolated. }\end{array}$ \\
\hline Yun et al., 2010 [8] & $\begin{array}{l}\text { Polyacrylonitrile } \\
\text { (PAN) }\end{array}$ & $\begin{array}{l}390 \\
420 \\
420\end{array}$ & $\begin{array}{l}\mathrm{BF}=0.235-0.356 \\
\mathrm{CF}=0.094-0.206 \\
\mathrm{NF}=0.051-0.174\end{array}$ & $\begin{array}{l}\text { BF stands for beaded nanofiber; CF: composite } \\
\text { particle nanofiber; NF: nanofibers. The highest value } \\
\text { of OF of BF is for } 300 \mathrm{~nm} \text { particles; the highest } \\
\text { values of OF of CF and NF are for } 20 \mathrm{~nm} \text { particles. } \\
U=5.3 \mathrm{~cm} / \mathrm{s}\end{array}$ \\
\hline Zhang et al., 2010 [9] & $\begin{array}{l}\text { Polyacrylonitrile } \\
\text { (PAN) }\end{array}$ & 200 & $\begin{array}{c}\text { PAN5 }=0.037 \\
\text { PAN15 }=0.023 \\
\text { PAN5x3 }=0.063 \\
\text { PAN15x2 }=0.025\end{array}$ & $\begin{array}{l}\text { "x3" stands for } 3 \text { layers of PAN nanofiber and "x2" } \\
\text { stands for } 2 \text { layers. } U=5.3 \mathrm{~cm} / \mathrm{s}\end{array}$ \\
\hline Leung et al., 2010 [7] & $\begin{array}{l}\text { Polyethylene oxide } \\
\text { (PEO) }\end{array}$ & 208 & $\begin{aligned} \mathrm{N} 9 \mathrm{~S} & =0.015 \\
\mathrm{~N} 8 \mathrm{~S} & =0.016 \\
\mathrm{~N} 7 \mathrm{~S} & =0.017 \\
\mathrm{~N} 6 \mathrm{~S} & =0.019 \\
\mathrm{~N} 5 \mathrm{~S} & =0.024 \\
\mathrm{~N} 1 \mathrm{~S} & =0.045\end{aligned}$ & $\begin{array}{l}\text { QF for } U=5 \mathrm{~cm} / \mathrm{s} \text { was interpolated; } \\
\text { N1S had the lowest filtration efficiency and pressure } \\
\text { drop, while N9S had the highest filtration efficiency } \\
\text { and pressure drop. }\end{array}$ \\
\hline Hung and Leung, 2011 [22] & PA 6 & $\begin{array}{c}94 \\
185 \\
185 \\
185 \\
185 \\
220\end{array}$ & $\begin{array}{c}S=0.039 \\
M 1=0.037 \\
M 2=0.029 \\
M 3=0.027 \\
M 4=0.022 \\
L=0.022\end{array}$ & QF for $U=5 \mathrm{~cm} / \mathrm{s}$ was interpolated \\
\hline
\end{tabular}

of polyamide fibers depended not only on the collection time, but also on the tip-to-collector distance. The comparative analysis of the polyamide 6 and polyamide $6 / 6$ nanofiber characteristics revealed that the polyamide $6 / 6$ had higher potential to be used in air filtration applications. Based on the results of complex experimental design data, response surface plots for obtaining nanofiber media with desirable polyamide 6 and polyamide 6/6/fiber diameter and basis weight characteristics were derived and presented.

The filtration properties of PA nanofiber media fabricated according to the modelling results showed that nanofiber filter media produced from PA6/6 8\% (w/vol) solution have the highest filtration efficiency (PA6/6_30 = 84.9-90.9\%). The highest quality factor $\left(0.0486-0.0749 \mathrm{~Pa}^{-1}\right)$ was received for PA6/6_10 nanofiber filter media and at the same time it exceeded larger part of filter quality factors obtained by other authors for most penetrating particle size $(300 \mathrm{~nm})$. This supports the assumption that principal component analysis modelling could be applied in obtaining nanofiber media with characteristics suitable for air filtration applications. The nanofiber media characteristics such as fiber diameter and basis weight can be predicted in advance resulting in fabrication of filter media.

\section{Conflict of Interests}

The authors declare that there is no conflict of interests regarding the publication of this paper.

\section{References}

[1] N. Bhardwaj and S. C. Kundu, "Electrospinning: a fascinating fiber fabrication technique," Biotechnology Advances, vol. 28, no. 3, pp. 325-347, 2010.

[2] S. Mahalingam and M. Edirisinghe, "Forming of polymer nanofibers by a pressurised gyration process," Macromolecular Rapid Communications, vol. 34, no. 14, pp. 1134-1139, 2013.

[3] G. Ward, "Nanofibres: media at the nanoscale," Filtration and Separation, vol. 42, no. 7, pp. 22-24, 2005.

[4] S. Petrik and M. Maly, "Production nozzle-less electrospinning nanofiber technology," in Proceedings of the Fall MRS Symposium, pp. 79-90, Boston, Mass, USA, November-December 2009.

[5] Q. P. Pham, U. Sharma, and A. G. Mikos, "Electrospinning of polymeric nanofibers for tissue engineering applications: a review," Tissue Engineering, vol. 12, no. 5, pp. 1197-1211, 2006. 
[6] X. Wang, H. Niu, X. Wang, and T. Lin, "Needleless electrospinning of uniform nanofibers using spiral coil spinnerets," Journal of Nanomaterials, vol. 2012, Article ID 785920, 9 pages, 2012.

[7] W. W.-F. Leung, C.-H. Hung, and P.-T. Yuen, "Effect of face velocity, nanofiber packing density and thickness on filtration performance of filters with nanofibers coated on a substrate," Separation and Purification Technology, vol. 71, no. 1, pp. 30-37, 2010.

[8] K. M. Yun, A. B. Suryamas, F. Iskandar, L. Bao, H. Niinuma, and K. Okuyama, "Morphology optimization of polymer nanofiber for applications in aerosol particle filtration," Separation and Purification Technology, vol. 75, no. 3, pp. 340-345, 2010.

[9] Q. Zhang, J. Welch, H. Park, C.-Y. Wu, W. Sigmund, and J. C. M. Marijnissen, "Improvement in nanofiber filtration by multiple thin layers of nanofiber mats," Journal of Aerosol Science, vol. 41, no. 2, pp. 230-236, 2010.

[10] W. C. Hinds, Aerosol Technology: Properties, Behavior, and Measurement of Airborne Particles, John Wiley \& Sons, New York, NY, USA, 2nd edition, 1998.

[11] P. Heikkilä, A. Taipale, M. Lehtimäki, and A. Harlin, "Electrospinning of polyamides with different chain compositions for filtration application," Polymer Engineering and Science, vol. 48, no. 6, pp. 1168-1176, 2008.

[12] A. Podgórski, A. Bałazy, and L. Gradoń, "Application of nanofibers to improve the filtration efficiency of the most penetrating aerosol particles in fibrous filters," Chemical Engineering Science, vol. 61, no. 20, pp. 6804-6815, 2006.

[13] J. Wang, S. C. Kim, and D. Y. H. Pui, "Investigation of the figure of merit for filters with a single nanofiber layer on a substrate," Journal of Aerosol Science, vol. 39, no. 4, pp. 323-334, 2008.

[14] M. Chowdhury and G. Stylios, "Effect of experimental parameters on the morphology of electrospun nylon 6 fibres," International Journal of Basic \& Applied Sciences, vol. 10, no. 6, pp. 70-78, 2010.

[15] B. de Schoenmaker, L. van der Schueren, Ö. Ceylan, and K. de Clerck, "Electrospun polyamide 4.6 nanofibrous nonwovens: parameter study and characterization," Journal of Nanomaterials, vol. 2012, Article ID 860654, 9 pages, 2012.

[16] L. M. Guerrini, M. C. Branciforti, T. Canova, and R. E. S. Bretas, "Electrospinning and characterization of polyamide 66 nanofibers with different molecular weights," Materials Research, vol. 12, no. 2, pp. 181-190, 2009.

[17] P. Heikkilä and A. Harlin, "Parameter study of electrospinning of polyamide-6," European Polymer Journal, vol. 44, no. 10, pp. 3067-3079, 2008.

[18] S. Lingaiah, K. Shivakumar, and R. Sadler, "Electrospinning of nylon-66 polymer nanofabrics," in Proceedings of the 49th AIAA/ASME/ASCE/AHS/ASC Structures, Structural Dynamics, and Materials Conference, Schaumburg, Ill, USA, April 2008.

[19] B. Pant, H. R. Pant, D. R. Pandeya et al., "Characterization and antibacterial properties of Ag NPs loaded nylon-6 nanocomposite prepared by one-step electrospinning process," Colloids and Surfaces A: Physicochemical and Engineering Aspects, vol. 395, pp. 94-99, 2012.

[20] H. R. Pant, M. P. Bajgai, K. T. Nam et al., "Electrospun nylon6 spider-net like nanofiber mat containing $\mathrm{TiO}_{2}$ nanoparticles: a multifunctional nanocomposite textile material," Journal of Hazardous Materials, vol. 185, no. 1, pp. 124-130, 2011.

[21] H. R. Pant, M. P. Bajgai, C. Yi et al., "Effect of successive electrospinning and the strength of hydrogen bond on the morphology of electrospun nylon-6 nanofibers," Colloids and
Surfaces A: Physicochemical and Engineering Aspects, vol. 370, no. 1-3, pp. 87-94, 2010.

[22] C.-H. Hung and W. W.-F. Leung, "Filtration of nano-aerosol using nanofiber filter under low Peclet number and transitional flow regime," Separation and Purification Technology, vol. 79, no. 1, pp. 34-42, 2011.

[23] S. Zhang, W. S. Shim, and J. Kim, "Design of ultra-fine nonwovens via electrospinning of nylon 6: spinning parameters and filtration efficiency," Materials and Design, vol. 30, no. 9, pp. 3659-3666, 2009.

[24] I. M. Hutten, Handbook of Nonwoven Filter Media, Elsevier Science and Technology Books, 2007.

[25] M. Alonso, M. I. Martin, and F. J. Alguacil, "The measurement of charging efficiencies and losses of aerosol nanoparticles in a corona charger," Journal of Electrostatics, vol. 64, no. 3-4, pp. 203-214, 2006.

[26] N. A. M. Barakat, M. A. Kanjwal, F. A. Sheikh, and H. Y. Kim, "Spider-net within the N6, PVA and PU electrospun nanofiber mats using salt addition: novel strategy in the electrospinning process," Polymer, vol. 50, no. 18, pp. 4389-4396, 2009.

[27] V. Beachley and X. Wen, "Effect of electrospinning parameters on the nanofiber diameter and length," Materials Science and Engineering C: Materials for Biological Applications, vol. 29, no. 3, pp. 663-668, 2009.

[28] J. Wang, S. C. Kim, and D. Y. H. Pui, "Figure of merit of composite filters with micrometer and nanometer fibers," Aerosol Science and Technology, vol. 42, no. 9, pp. 722-728, 2008. 

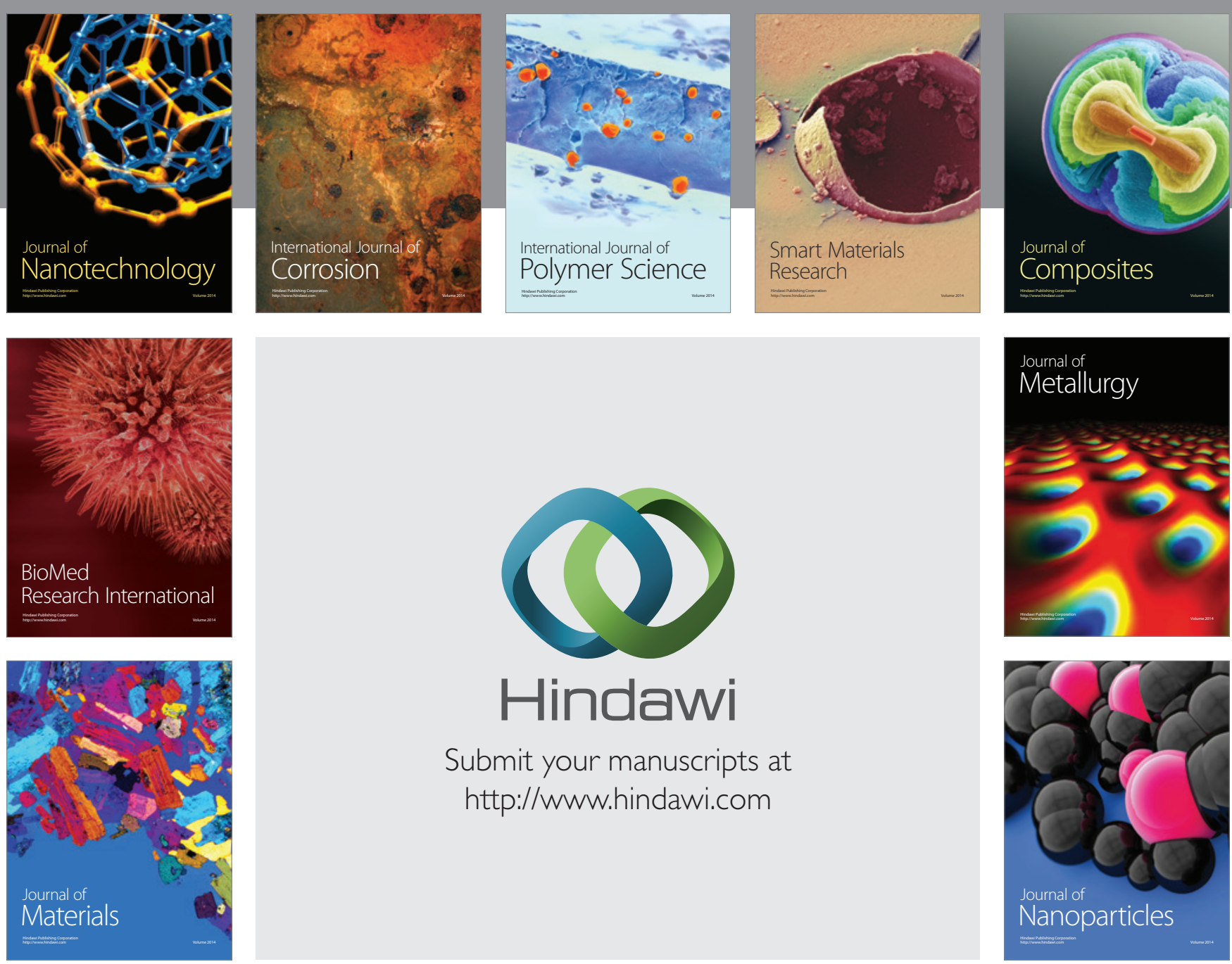

Submit your manuscripts at http://www.hindawi.com
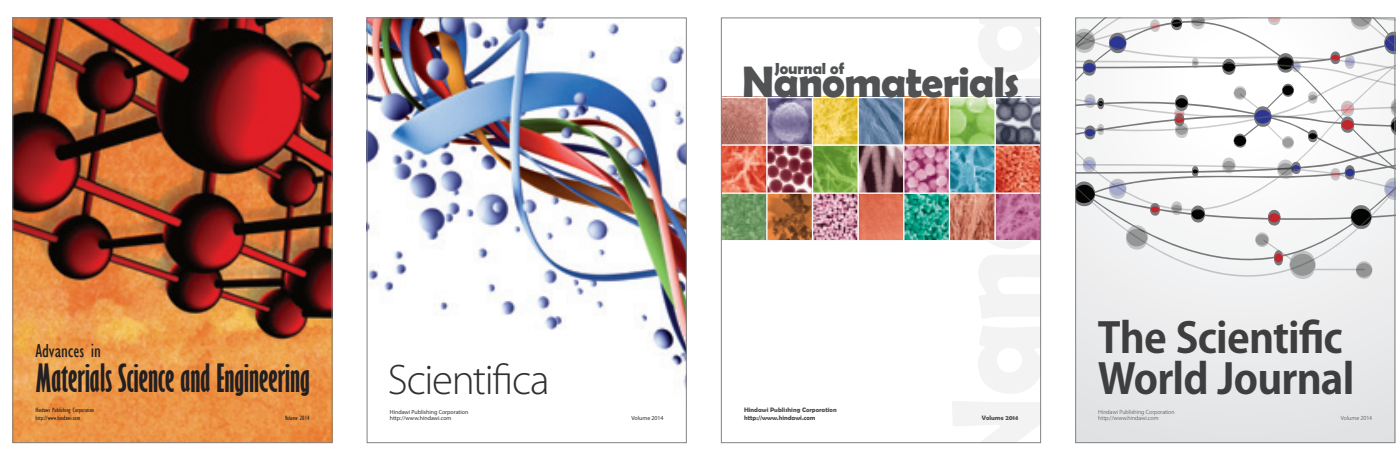

\section{The Scientific World Journal}
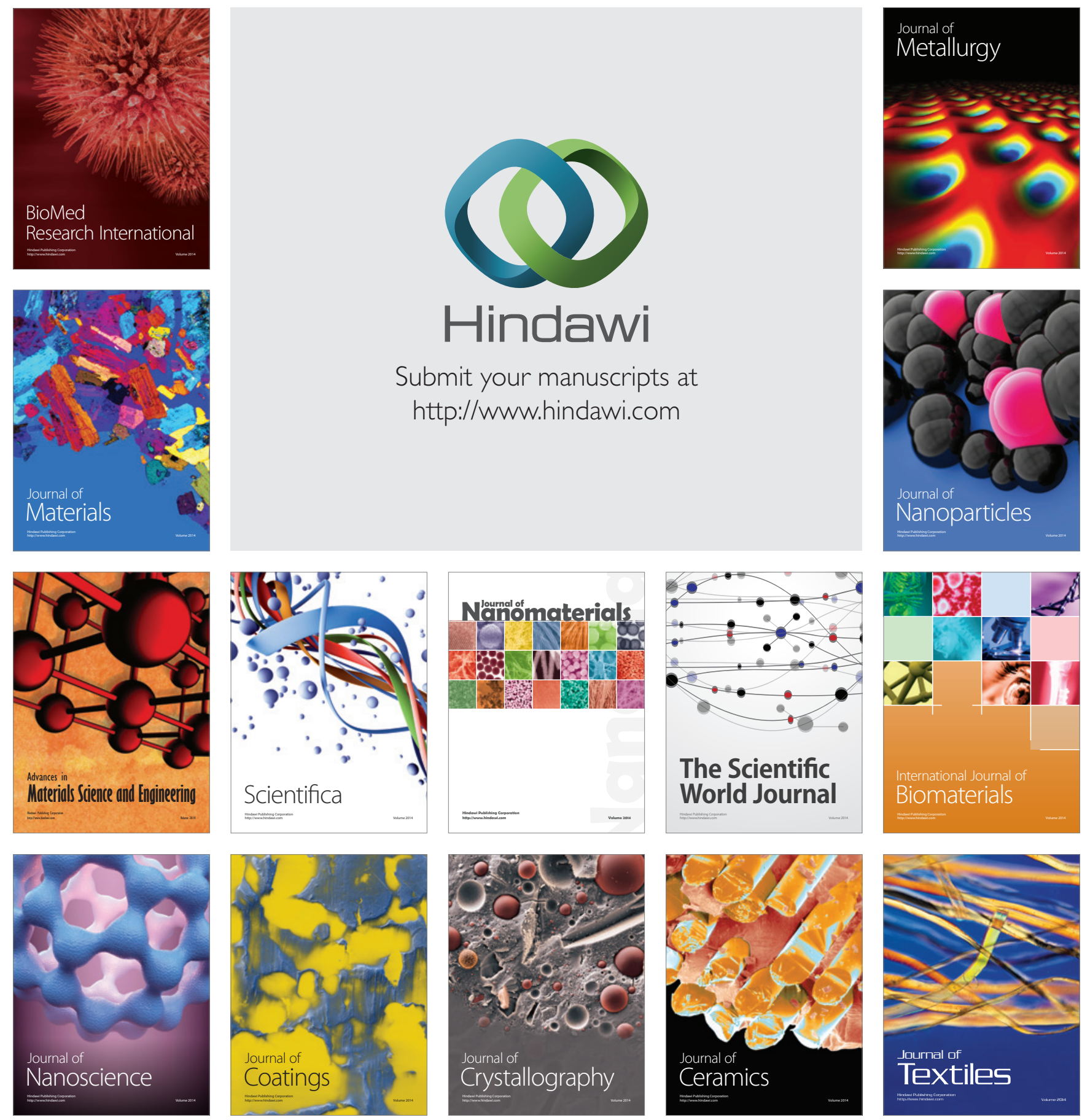